\title{
Característica clínicas e radiológicas de pacientes com pneumonia por Mycoplasma pneumoniae internados no Hospital Infantil Sabará
}

Chiba SM, Gomes, CET, Sawamura MVY, Almeida LR, Suzuki L, Ionemoto HF.

\section{Resumo}

Introdução: Mycoplasma pneumoniae (Mp) é uma causa comum de pneumonia adquirida na comunidade, relatada em 10 a 40\% dos casos especialmente na idade escolar e adultos jovens. Mycoplasma pneumoniae (Mp), atinge o trato respiratório superior e inferior, com uma variedade de manifestações pulmonares e extra pulmonares. Afeta crianças de todas as faixas etárias, mas as manifestações clínicas variam de acôrdo com a idade, desde infecções assintómaticas a pneumonias fatais. Objetivos: Descrever as principais manifestações clínicas, laboratoriais e aspectos radiológicos da pneumonia por Mp em crianças internadas no Hospital Sabara no periodo de julho a dezembro/2013. Material e Métodos: Levantamento retrospectivo de prontuarios com dados clínicos e laboratoriais e radiografias de torax dos pacientes internados com diagnóstico de pneumonia por Mycoplasma pneumoniae confirmado através de metodo sorologico (ELISA). Os raio $\mathrm{x}$ de torax foram avaliados separadamente por 2 radiologistas( especialista em radiologia pediátrica e radiologia torácica). As alterações do parênquima pulmonar foram classificadas em três grupos: 1) Espessamento intersticial peribroncovascular 2) opacidade/ consolidação 3) associação entre os dois padrões. Metodologia estatística As características demográficas e clínicas foram analisadas descritivamente. Para as variáveis de natureza categórica foram apresentadas frequências absolutas e relativas e para as variáveis de natureza numérica calculadas média, desvio padrão, quartis, mínimo e máximo. O software estatístico utilizado para as análises foi o SPSS 20.0. Resultados: Foram analisados os dados de 29 pacientes, com sorologia IGM positivo para mycoplasma pneumoniae, dos quais 17 (58,6\%) eram do sexo feminino. A média da idade dos pacientes foi de 6,6 anos ( $\mathrm{DP}=3,4$ anos), sendo observada uma idade mínima de 2 anos e máxima de 16 anos. A mediana das idades foi de 6 anos. Na admissão hospitalar todos os pacientes deste estudo apresentaram tosse e 79,3\% tiveram febre. A media da duração da tosse foi de 7,0 (DP=5,9) dias e 4,3 dias de febre ( $\mathrm{DP}=3,5)$. Os sintomas associados na admissão estiveram presentes em $62 \%$, tais como coriza, cefaleia, vomitos, dor abdominal e urticária. Mais de 50\% tinham historia de uso previo de antibiótico. Em média, os pacientes permaneceram 6,2 dias ( $\mathrm{DP}=3,4$ dias) de internação. $\mathrm{O}$ hemograma inicial demonstrou uma 
media da hemoglobina de 12,5g ( $\mathrm{DP}=1,2)$ e leucocitos 12.093,1(DP=5269,0) com predomínio de neutrofilia. Na analise radiológica 51,7\% dos pacientes apresentaram infiltrado intersticial e opacidades/consolidação simultaneamente, somente opacidade/consolidação em 31\% , infiltrado intersticial em $17,2 \%$ e derrame pleural em $13.8 \%$. Nota-se que no total foram observadas $82,8 \%$ de casos com opacidades/consolidação. Com relação à distribuição verificou-se que 58,6\% tiveram alteração bilateral e 31,0\%, focal. conclusão: A faixa predominante foi em escolares. Os sintomas mais importantes foram febre e tosse prolongada. O hemograma em geral não foi infeccioso. $\mathrm{O}$ achado radiológico mais comum foi opacidade e consolidação e diferente dos demais trabalhos o acometimento radiológico foi bilateral. Necessitamos de mais estudos para avaliar a pneumonia por Mp em crianças menores de 5 anos. A frequencia da pneumonia por Mycoplasma pneumoniae é ainda subestimada na pratica clínica por causa da falta de um quadro clínico especifico e o exame diagnóstico que necessita de um método sorológico ou reação em cadeia polimerase (PCR) nem sempre disponíveis nos serviços de saude em nosso meio.

Palavras chave: mycoplasma pneumoniae; pneumonia; tosse

Chiba SM, Gomes, CET, Sawamura MVY, Almeida LR, Suzuki L, Ionemoto HF. “Característica clínicas e radiológicas de pacientes com pneumonia por Mycoplasma pneumoniae internados no Hospital Infantil Sabará", in Anais do 2‥ Congresso Internacional Sabará de Especialidades Pediátricas

English Title: Clinical and radiological characteristics of patients with Mycoplasma pneumoniae pneumonia hospitalized in the Children's Hospital Sabará

Abstract:.Mycoplasma pneumoniae (Mp) is a common cause of community-acquired pneumonia. It has been reported in $10-40 \%$ of cases especially in school age and young adults. Mycoplasma pneumoniae (Mp), accounts the upper and lower respiratory tract, with a variety of pulmonary and extra pulmonary manifestations. Affects children of all age groups, but the clinical manifestations vary with age, from asymptomatic infections to fatal pneumonia. The objectives this study was to describe the main clinical and laboratory manifestations and radiological aspects of Mycoplasma pnemoniae pneumonia in hospitalized children.

\section{Referencias}

1). Atkinson TP, Balish MF, Waites KB. Epidemiology, clinical manifestations, pathogenesis and laboratory detection of Mycoplasma pneumoniae infections. FEMS Microbiol Rev 2008;32:956-73.

2). Lee KY. Pediatric respiratory infections by Mycoplasma pneumoniae. Expert Rev Anti Infect Ther 2008;6:509-21. 
3) Sørensen CM, Schønning K, Rosenfeldt V. Clinical characteristics of children with Mycoplasma pneumonia infection hospitalized during the Danish 2010-2012 epidemic.Dan Med J 2013; 60 : A4632

4) Youn YS, Lee KY. Mycoplasma pneumoniae pneumonia in children. Korean J Pediatr 2012;55:42-47 